INFLAMMATORY BOWEL DISEASE

\title{
A randomised, controlled, double blind, escalating dose study of alicaforsen enema in active ulcerative colitis
}

\author{
S J H van Deventer, J A Tami, M K Wedel, European Colitis Study Group
}

Gut 2004;53:1646-1651. doi: 10.1136/gut.2003.036160

See end of article for authors' affiliations

Correspondence to:

Professor S J H van

Deventer, Academisch

Medisch Centrum, Room

G2, 129, Poli

Inglammatoire

Darmzietkten

Meibergdreef 9, NL 1105

AZ, Amsterdam Ziodoost

the Netherlands;

S.J.vanDeventer@

amc.uva.nl

Revised version received

2 April 2004

Accepted for publication

3 April 2004
Objective: To evaluate the safety and efficacy of an enema formulation of alicaforsen, an antisense inhibitor of intercellular adhesion molecule, after 1, 3, and 6 months.

Methods: This was a randomised, placebo controlled, double blind, escalating dose multicentre study in 40 patients with mild to moderately active distal ulcerative colitis (disease activity index (DAl) 4-10).

Patients were assigned to four dosing cohorts of 10 patients each (eight active, two placebo). Each patient received $60 \mathrm{ml}$ of alicaforsen enema $(0.1,0.5,2$, or $4 \mathrm{mg} / \mathrm{ml}$ or placebo) once daily for 28 consecutive days. Safety and efficacy (DAl and clinical activity index) scores were evaluated up to six months after initiation of dosing.

Results: At day 29, alicaforsen enema resulted in dose dependent improvement in DAl (overall $p=0.003$ ). Alicaforsen $4 \mathrm{mg} / \mathrm{ml}$ improved DAl by $70 \%$ compared with the placebo response of $28 \%(p=0.004)$. Alicaforsen 2 and $4 \mathrm{mg} / \mathrm{ml}$ improved DAl status by $72 \%$ and $68 \%$ compared with a placebo response of $11.5 \%$ at month 3 ( $p=0.016$ and 0.021 , respectively). Specifically, DAl improved from 5.6 to 1.6 and from 6.3 to 2.5 in the 2 and $4 \mathrm{mg} / \mathrm{ml}$ groups compared with placebo ( 7.5 to 6.1 ). None of the patients in the $4 \mathrm{mg} / \mathrm{ml}$ group compared with $4 / 8$ placebo patients required additional medical or surgical intervention over baseline during the six month period after starting the enema treatment. The safety profile was favourable.

Conclusions: Alicaforsen enema showed promising acute and long term benefit in patients with mild to moderate descending ulcerative colitis. Alicaforsen enemas had a favourable safety profile. These findings require verification in larger randomised controlled clinical trials.
M esalamine, administered orally or as an enema, is the mainstay of therapy for patients with mild to moderate ulcerative colitis. Meta-analyses of therapeutic trials in patients with left sided colitis or proctitis have reported that topical mesalamine treatment is superior to oral mesalamine, results in higher remission rates than rectal corticosteroid administration, and is safe. ${ }^{12}$ Hence topical mesalamine treatment is currently the preferred therapy for patients with mild to moderate left sided ulcerative colitis. However, $50-60 \%$ of patients treated with mesalamine enema do not achieve complete endoscopic remission within 4-8 weeks of therapy. A smaller fraction of patients are completely resistant to mesalamine therapy necessitating treatment with corticosteroids or immunosuppressives, both of which are associated with significant toxicity. Therefore, there is a continuing need for new effective and non-toxic therapies for left sided ulcerative colitis.

Recruitment of neutrophils from the intravascular space into local sites of inflammation is a multistep process resulting from selectin dependent slowing of the neutrophil on the endothelial surface ("rolling") and activation of neutrophil $\beta$-integrins, in particular CD1 lb/CD18 (Mac-1), by platelet activating factor and CXC chemokines ( $\alpha$ chemokines that have one amino acid residue separating the first two conserved cysteine residues), a process which leads to firm adherence.

Intercellular adhesion molecule 1 (ICAM-1), a member of the immunoglobulin superfamily, is an inducible transmembrane glycoprotein that is constitutively expressed at low levels on vascular endothelial cells and a subset of leucocytes. ${ }^{3-5}$ In response to proinflammatory mediators, many cell types, including colonic epithelium, upregulate expression of ICAM-1 on their surface. The primary counterligands for ICAM-1 are the $\beta_{2}$-integrins, leucocyte function associated antigen 1, and Mac-1, all expressed on leucocytes. ${ }^{67}$ ICAM-1 serves multiple functions in the propagation of inflammatory processes, including facilitating roles in leucocyte activation and migration from the intravascular space in response to inflammatory stimuli. ${ }^{8-10}$ Several studies have reported increased ICAM-1 expression within the inflamed gut mucosa as well as increased circulating concentrations of soluble ICAM-1 in experimental colitis and in inflammatory bowel disease. ${ }^{11}$ The functional importance of ICAM-1 expression for recruitment of neutrophils was demonstrated in intervention studies in experimental colitis that reported therapeutic benefit from administration of either antibodies or antisense oligonucleotides targeting ICAM- $1 .{ }^{12}{ }^{13}$ These observations indicate that ICAM-1 inhibition is an attractive target for the development of new agents for ulcerative colitis.

Alicaforsen (ISIS 2302; Isis Pharmaceuticals, Inc., Carlsbad, California, USA) is a 20 base phosphorothioate oligodeoxynucleotide antisense molecule designed to downregulate messenger RNA for ICAM-1. Antisense inhibition of ICAM-1 via retention enema is a means of achieving topical drug delivery to the involved colon while minimising systemic bioavailability. This study is the first clinical evaluation of alicaforsen enema. The objective of the study was to evaluate the acute and long term safety and efficacy of four different doses of alicaforsen enema administered once a day for 28 consecutive days.

Abbreviations: ICAM-1, intercellular adhesion molecule 1; DAI, disease activity index; CAl, clinical activity index; LLOQ, lower limit of quantitation 


\section{MATERIALS AND METHODS}

Institutional ethics committees approved the protocol and consent forms, and the study was conducted in accordance with local and national regulatory guidelines. Candidates for the study were patients with active distal ulcerative colitis extending $5-50 \mathrm{~cm}$ from the anal verge (confirmed by endoscopy) who were at least 18 years old, had a disease activity index (DAI) $)^{14}$ score of 3-10, and were receiving either a stable oral dose of 5-ASA (1500-3000 mg) for at least two months prior to the study or no background oral therapy (except antidiarrhoeals and analgesics) for two months prior to the study.

Protocol exclusions included: current use of other treatments for ulcerative colitis (for example, steroids, 5-ASA enema, immunosuppressants) except oral 5-ASA, antidiarrhoeal, and analgesic agents during the study dosing period; concomitant use of non-steroidal anti-inflammatory drugs or antibiotics effective against gastrointestinal organisms; complications of ulcerative colitis such as bowel stricture, toxic megacolon, or colon carcinoma; a history of colonic resection; a positive stool culture for enteric pathogens or $C$ difficile toxin in stool; other infectious, ischaemic or immunological diseases with gastrointestinal involvement; active infection; active malignancy; uncontrolled haematological, hepatic, renal, pulmonary, or cardiovascular disease; severe infection or a major surgical procedure within one month prior to dosing; and significant laboratory abnormalities.

A central pharmacy assigned patients sequentially to four cohorts of 10 patients each (eight study drug, two placebo) and randomised each patient to receive study drug or placebo. Each patient kit of enema bottles was labelled with a unique reference number and a scratch off code break to blind the investigators, study monitors, and patients to treatment assignment.

The first cohort received $0.1 \mathrm{mg} / \mathrm{ml}$ of alicaforsen enema, the second cohort $0.5 \mathrm{mg} / \mathrm{ml}$, the third cohort $2 \mathrm{mg} / \mathrm{ml}$, and the fourth cohort $4 \mathrm{mg} / \mathrm{ml}$. Each dosing cohort contained two patients who received placebo enema. Progression to the next higher dosing cohort could not occur until all members of the previous cohort had completed at least two weeks of dosing

Table 1 Disease activity index (Schroeder and colleagues $^{14}$ )

\begin{tabular}{ll}
\hline Activity & Index No \\
\hline Stool frequency & 0 \\
Healthy* & 1 \\
$1-2$ stools per day > healthy & 2 \\
$3-4$ stools per day > healthy & 3 \\
$>4$ stools per day > healthy & 0 \\
Rectal bleeding & 1 \\
None & 2 \\
Streaks of blood with stool less than half the time & 3 \\
Obvious blood with stool most of the time & 0 \\
Blood alone passed & 1 \\
Endoscopic appearance & \\
Normal or inactive disease & \\
Mild (erythema, decreased vascular pattern, mild \\
friability)
\end{tabular}

without significant toxicity. Each patient self administered a $60 \mathrm{ml}$ retention enema in the evening for 28 consecutive days. Patients who responded to treatment were followed until recurrence or six months after treatment had begun. Patients who did not respond to treatment were followed for safety data until two months after dosing started and were allowed to exit the trial to pursue alternative treatment. Efficacy was measured by DAI (table 1) and clinical activity index (CAI) (table 2)..$^{14} 15$

The intent to treat population $(\mathrm{n}=40)$ was used for analysis of the primary efficacy end points, DAI and CAI. DAI is a composite score based on the daily number of stools, visible blood in stool, appearance of the colonic mucosa at endoscopy, and the investigator's global assessment. DAI was assessed at baseline (day 1), day 29, and at months 3 and 6 . CAI is a composite score based on the number of daily stools, nocturnal diarrhoea, visible blood in stool, faecal incontinence, abdominal cramping, general well being, abdominal tenderness, and the need for antidiarrhoeal medication. CAI is an unvalidated clinical scoring system for the severity of symptoms and quality of life related to ulcerative colitis. Because it is entirely subjective and provided for the collection of more frequent data points, this non-invasive and subjective assessment was included as an exploratory end point. CAI was assessed at screening, baseline (day 1), days $8,15,22$, and 29, and again at months 2, 3, 4, 5, and 6 . Secondary efficacy end points include the four individual components of the DAI score and alicaforsen drug concentration measured in mucosal biopsy samples obtained by

Table 2 Clinical activity index (modified from Truelove and Witts ${ }^{15}$ )

\begin{tabular}{|c|c|}
\hline Symptom & Index No \\
\hline \multicolumn{2}{|l|}{ Diarrhoea (No of daily stools) } \\
\hline $0-2$ & 0 \\
\hline 3 or 4 & 1 \\
\hline 5 or 6 & 2 \\
\hline $7-9$ & 3 \\
\hline 10 or more & 4 \\
\hline \multicolumn{2}{|l|}{ Nocturnal diarrhoea } \\
\hline No & 0 \\
\hline Yes & 1 \\
\hline \multicolumn{2}{|c|}{ Visible blood in stool (\% of movements) } \\
\hline 0 & 0 \\
\hline$<50$ & 1 \\
\hline$\geqslant 50$ & 2 \\
\hline 100 & 3 \\
\hline \multicolumn{2}{|l|}{ Faecal incontinence } \\
\hline No & 0 \\
\hline Yes & 1 \\
\hline \multicolumn{2}{|l|}{ Abdominal pain or cramping } \\
\hline None & 0 \\
\hline Mild & 1 \\
\hline Moderate & 2 \\
\hline Severe & 3 \\
\hline \multicolumn{2}{|l|}{ General well being } \\
\hline Perfect & 0 \\
\hline Very good & 1 \\
\hline Good & 2 \\
\hline Average & 3 \\
\hline Poor & 4 \\
\hline Terrible & 5 \\
\hline \multicolumn{2}{|l|}{ Abdominal tenderness } \\
\hline None & 0 \\
\hline Mild or localised & 1 \\
\hline Mild to moderate and diffuse & 2 \\
\hline Severe or rebound & 3 \\
\hline \multicolumn{2}{|l|}{ Need for antidiarrhoeal drugs } \\
\hline No & 0 \\
\hline Yes & 1 \\
\hline \multicolumn{2}{|c|}{$\begin{array}{l}\text { The clinical activity index score was based on average ratings over the } \\
\text { seven days prior to the visit day, as recorded in a diary card by the } \\
\text { patient. }\end{array}$} \\
\hline
\end{tabular}


duplicate pinch biopsies of affected bowel mucosa at sigmoidoscopy prior to dosing on day 1 and again within 12 hours of the last dose administration on day 29.

Patients were evaluated for adverse events throughout the six month period. Coagulation, chemistry, haematology, and urinalysis assessments were made through to day 29. Twelve lead electrocardiograms were obtained at screening and on day 29, and vital signs were monitored throughout.

Biopsies of affected bowel mucosa were obtained during endoscopy prior to dosing on day 1 and within 12 hours of the last dose on day 29 for evaluation of tissue pharmacokinetics. The biopsy was snap frozen in liquid nitrogen, stored at $-70^{\circ} \mathrm{C}$, and shipped on dry ice to Isis Pharmaceuticals, Inc. (Carlsbad, California, USA) for analysis of ISIS 2302 concentrations in biopsies. Briefly, samples were weighed and digested using protease K prior to analysis for ISIS 2302 concentration using a hybridisation ELISA method. ${ }^{16}$ Sample concentrations were determined by interpolation from the standard curve prepared in drug free porcine intestinal mucosa, which was fit using a log-log function. The quantification range was $0.271-54.3 \mathrm{ng}$ of ISIS $2302 \mathrm{in}$ biopsies (or 54.2-1086 ng/g for a $5 \mathrm{mg}$ biopsy), with the low end of this range defining the lower limit of quantitation (LLOQ). Because the weight of biopsy samples was generally in the range $2-10 \mathrm{mg}$, the corresponding LOQ concentration was in the range 135.5-27.1 ng/g. Samples with concentrations previously observed or expected to be above the upper level of quantitation were diluted within the range using a suitable volume of drug free porcine intestinal mucosa digest prior to analysis. Cross reactivity of the assay was less than $0.22 \%$ for $3^{\prime}$-metabolites.

A generalised linear model was used to analyse the per cent change from baseline for each of the primary and secondary efficacy end points in all five treatment groups simultaneously to determine whether there was a dose-response relationship at day 29 (month 1), as defined in the study protocol. DAI and CAI scores and change in DAI and CAI scores at each visit were summarised by dose level using descriptive statistics. Pairwise inferential statistical comparisons of per cent change from baseline were performed at subsequent time points to determine the duration of response to study drug. No adjustments were made for multiple comparisons because of the small number of planned patients enrolled in this exploratory study.

\section{RESULTS}

\section{Patient enrolment and disposition}

Forty five patients were screened for eligibility, five of whom were excluded. Reasons for screen failure included abnormal screening laboratories $(n=2)$, refusal to participate after signing informed consent $(\mathrm{n}=2)$, and one patient experienced a disease flare prior to dosing and withdrew from the study. Forty patients with mild to moderately active distal ulcerative colitis were enrolled, randomised, and followed for up to six months by 11 European investigators between February 2000 and July 2001. All 40 patients were dosed: $39 / 40$ and $24 / 40$ patients completed the study through to months 2 and 6 , respectively. Sixteen patients did not complete the study, 15 due to worsening disease and one patient $(0.1 \mathrm{mg} / \mathrm{ml})$ for an adverse event (increased liver enzymes possibly related to the study drug).

\section{Demographics and baseline characteristics}

Patients were predominantly male $(24 / 40)$ and Caucasian (39/40). Mean ages were comparable between treatment groups (range 38.4-48.9 years). Heights and weights were generally within normal limits for sex and age. Duration of ulcerative colitis ranged from months to 24 years with mean values between treatment groups ranging from 3.6 to
8.8 years. Individual values for total number of centimetres of colitis involved colon ranged from 6 to $50 \mathrm{~cm}$, with mean values between treatment groups ranging from 22.5 to $33.1 \mathrm{~cm}$.

Thirty seven of 40 patients were on a stable dose of 5-ASA at the time of enrolment. Ulcerative colitis treatments administered prior to screening included 5-ASA alone (43\%, $17 / 40$ ), corticosteroids (oral, enema, or intravenous) without other immune suppressants $(45 \%, 18 / 40)$, and immune suppressants with or without corticosteroids $(10 \%, 4 / 40)$. None of the patients had undergone prior surgery for ulcerative colitis.

\section{Dosing}

Patient compliance with the dosing regimens was excellent. Only one patient received less than 27 of the 28 scheduled doses (one patient in the $0.1 \mathrm{mg} / \mathrm{ml}$ treatment group received only 10 doses due to non-compliance). Mean daily volume of study treatment administered was comparable between treatment groups (range of means 54.7-61.4 ml) and mean duration of enema retention (measured from time of enema until first stool passage) ranged from 7.4 to 10.3 hours

\section{Efficacy}

Alicaforsen enema caused both acute and long term improvement in DAI status (figs 1, 2). On day 29 there was dose dependent improvement in DAI status (overall $\mathrm{p}=0.003)$. The $4 \mathrm{mg} / \mathrm{ml}$ dose improved DAI status by $70 \%$ compared with a placebo response of $28 \%(\mathrm{p}=0.004)$. The $2 \mathrm{mg} / \mathrm{ml}$ dose showed a trend in improvement of DAI status by $45 \%(p=0.201)$. Finally, at month 3 , the 2 and $4 \mathrm{mg} / \mathrm{ml}$ doses improved DAI status by $72 \%$ and $68 \%$ compared with a placebo response of $11.5 \%(\mathrm{p}=0.016$ and 0.021 , respectively). The improvement in DAI continued until month $6(56 \%$ and $64 \%$ improvement for $2 \mathrm{mg} / \mathrm{ml}$ and $4 \mathrm{mg} / \mathrm{ml}$, respectively). The latter did not reach statistical significance, possibly because only those placebo patients with spontaneous improvement still remained in the study. In the $2 \mathrm{mg} / \mathrm{ml}$ cohort, only one patient had withdrawn from the study before six months. None of the $4 \mathrm{mg} / \mathrm{ml}$ patients withdrew.

Consistent with the composite DAI score, alicaforsen enema improved several individual components of the DAI score by day 29. There was a dose dependent decrease in rectal bleeding (overall $\mathrm{p}=0.047$ ). There was a dose dependent improvement in endoscopic appearance (overall $p=0.035$ ). Following four weeks of enema therapy, complete

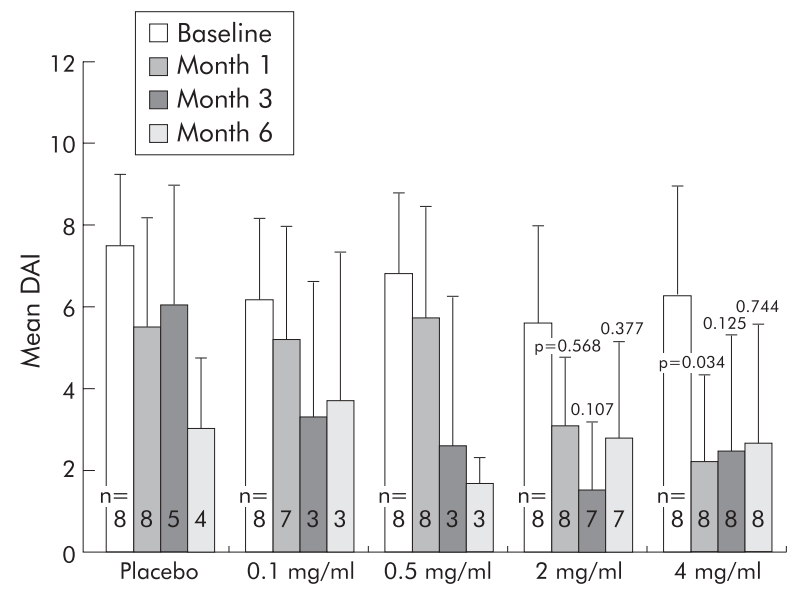

Figure 1 Mean absolute value for disease activity index (DAI) for each alicaforsen enema dose group is shown at each time point and compared with placebo. $p$ values are for change from baseline versus placebo. 


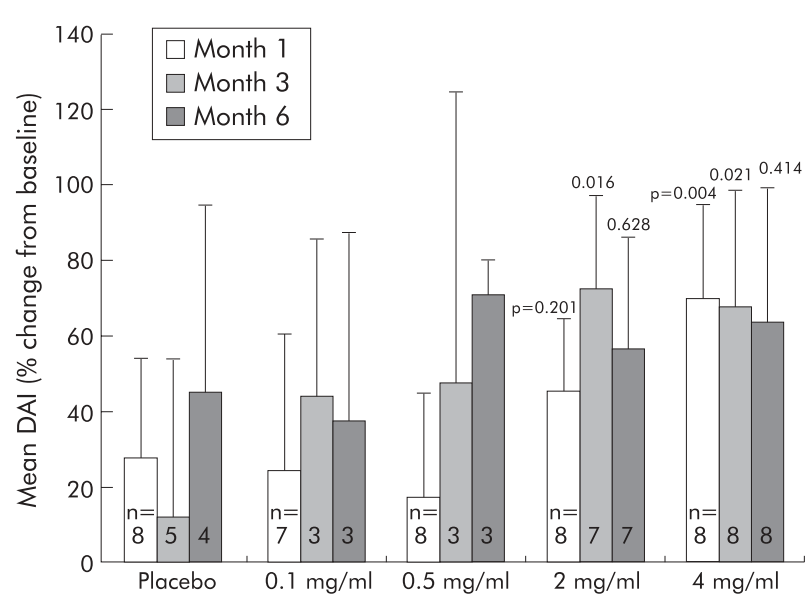

Figure 2 Mean per cent change in disease activity index (DAl) score from baseline for each alicaforsen enema dose group was compared with placebo at each time point. $p$ values versus placebo.

normalisation of endoscopy at three months (endoscopy score $=0)$ was noted in $56 \%(9 / 16)$ of patients receiving the $2 \mathrm{mg} / \mathrm{ml}$ or $4 \mathrm{mg} / \mathrm{ml}$ enema compared with $0 / 8$ in the placebo group. Finally, additional confirmation of the long term improvement in DAI status was demonstrated by the mean investigator's global assessment score. The 2 and $4 \mathrm{mg} / \mathrm{ml}$ doses resulted in a decrease in the investigator's global assessment score from 1.6 and 1.5 at baseline to 0.4 and 0.4 at month 3 , respectively ( $p=0.044$ and 0.043 ). Trend towards improvement in CAI score at day 29 was also seen (overall $\mathrm{p}=0.053)$.

Immunohistochemical evaluation of mucosal biopsies was performed. However, the results were not interpretable. The following factors are known to cause assay variability and may have influenced the results: (1) significant freeze artefact was present in the majority of tissue samples, (2) the subjective nature of assays that rely on tissue staining causes variability, (3) ICAM-1 expression is not homogenous in the colon and inability to precisely replicate the location of multiple biopsy samples in the same patient may have affected sequential concentration measurements, and (4) ICAM-1 concentration may vary over time.

More patients in the higher dosing groups completed the study than the lower dose and placebo groups, possibly suggesting drug effectiveness. In the 2 and $4 \mathrm{mg} / \mathrm{ml}$ treatment groups, $94 \%(15 / 16)$ of patients completed the study through to month 6 versus only $38 \%(9 / 24)$ in the placebo, 0.1 , and $0.5 \mathrm{mg} / \mathrm{ml}$ treatment groups. With the exception of one patient in the placebo, 0.1 , and $0.5 \mathrm{mg} / \mathrm{ml}$ treatment groups, the reason for not completing the study was worsening ulcerative colitis. In contrast, none of the patients in the $4 \mathrm{mg} / \mathrm{ml}$ group and only two patients in the $2 \mathrm{mg} / \mathrm{ml}$ group required another medication to treat ulcerative colitis in the six months after starting treatment with alicaforsen enema.

Administration of topical alicaforsen enema resulted in high tissue concentrations of drug in the colonic mucosa. Median concentrations of study drug measured in the pinch biopsy samples for the $0.1,0.5,2$, and $4 \mathrm{mg} / \mathrm{ml}$ treatment groups on day 29 were $0.48,2.40,2.36$, and $5.49 \mu \mathrm{g} / \mathrm{g}$, respectively.

\section{Safety}

Alicaforsen enema was well tolerated and there were no significant safety events. Adverse events were consistent with those commonly seen in this patient population. Adverse events (number of patients) reported in patients receiving placebo included pain (3), asthenia (1), nausea (1), and pharyngitis (1). Adverse events reported in two or more patients receiving alicaforsen enema included asthenia (3), infection (2), ulcerative colitis (2), and nausea (2). Three adverse events were judged to be serious and unrelated to the study drug using regulatory criteria. All required hospitalisation, two for exacerbation of ulcerative colitis (classified as unrelated to the study drug) and one for transfusion after presenting with exacerbation of symptoms and a haemoglobin level of $6.6 \mathrm{~g} / \mathrm{dl}$. The latter event was classified as having an unknown relationship to the study drug. In addition, dosing was stopped in one patient $(0.1 \mathrm{mg} / \mathrm{ml})$ due to an increase in liver function tests that was initially judged to be possibly related to the study drug. However, it was subsequently noted that this patient was non-compliant with dosing and the patient withdrew from the study.

No pattern of abnormality in any of the haematology, chemistry, or coagulation studies performed during the trial was observed. Patient vital signs assessed throughout the study period were unremarkable. No new abnormalities were seen on 12 lead EKGs taken on day 29.

\section{DISCUSSION}

The specific aim of this study was to investigate the potential efficacy and safety of topically administered alicaforsen in ulcerative colitis. We found that administration of alicaforsen in patients with mild to moderate ulcerative colitis caused a significant dose dependent reduction in disease activity. The $4 \mathrm{mg} / \mathrm{ml}$ dose of alicaforsen enema showed a statistically significant acute (day 29) improvement in DAI status. The 2 and $4 \mathrm{mg} / \mathrm{ml}$ doses of alicaforsen showed statistically significant long term (month 3 ) improvement in DAI status as well as a trend towards improvement in CAI. At the highest dose, DAI status on day 29 improved by $70 \%$ from baseline, from 6.3 to $2.2(\mathrm{p}=0.03) ; 2 / 8$ patients in the $2 \mathrm{mg} / \mathrm{kg}$ group and $3 / 8$ patients in the $4 \mathrm{mg} / \mathrm{kg}$ group had complete endoscopic remission on day 29. At three months, complete endoscopic remission was found in $4 / 8$ and $5 / 8$ patients ( $2 \mathrm{mg}$ and $4 \mathrm{mg} / \mathrm{kg}$ dose groups, respectively). Total treatment duration was four weeks. As this was an exploratory study with a small sample size, adjustments for multiple comparisons were not made. While the statistically significant results must be viewed cautiously because of the small sample size, they suggest drug activity in patients with mild to moderately active distal ulcerative colitis.

Consistent with the improvement in DAI, more patients completed the study through to month 6 in the 2 and $4 \mathrm{mg} / \mathrm{ml}$ treatment groups than in the 0.1 and $0.5 \mathrm{mg} / \mathrm{ml}$, and placebo treatment groups. None of the patients in the $4 \mathrm{mg} / \mathrm{ml}$ treatment group and only two patients in the $2 \mathrm{mg} / \mathrm{ml}$ treatment group (2/8) required additional medical or surgical intervention for ulcerative colitis during the treatment period through to month 6 . Patients who did not complete the study to month 6 generally did so because their colitis failed to respond or even worsened (15/16 cases), providing a correlation between the more objective DAI parameter and the clinical behaviour of patients and the physicians treating them. ISIS 2302 had a marked effect on DAI status and a lesser effect on CAI status. The reason for this difference is not entirely clear but a practical explanation is that DAI may provide a more objective and accurate measure of efficacy than CAI. The DAI score includes an objective endoscopic assessment of the colonic mucosa and also carries less subjectivity in its patient questionnaire components. By comparison, the CAI contains a number of subjective questions in its patient questionnaire and these questions also carry a great degree of subjectivity in their grading. In addition, while the 
results of the DAI and CAI differed, both showed positive trends.

Alicaforsen enema was well tolerated and there were no safety issues. Adverse events reported were consistent with those commonly seen in this patient population. There was no obvious or consistent relationship between alicaforsen enema and any adverse event.

Infiltration of neutrophils into the colonic mucosa is a principal event in the pathophysiology of ulcerative colitis. A variety of observations support a pivotal role for ICAM-1 in this process. Sonicated colonic tissue from ulcerative colitis patients contains higher concentrations of ICAM- 1 relative to controls. ICAM-1 significantly increases during active ulcerative colitis compared with ulcerative colitis in remission and increasing ICAM-1 concentration correlates with increasing disease activity. ${ }^{17}$ Ultrastructural studies of colonic biopsies from ulcerative colitis patients and control patients have demonstrated ICAM labelling on basal endothelial cell membranes, on macrophages, and on plasma cells in active ulcerative colitis, suggesting that ICAM-1 is involved in the entire leucocyte infiltration process and not simply in the early extravasation phase. ${ }^{18}$ Therefore, cell adhesion molecules, and ICAM- 1 in particular, appear to play a critical role in the migration of leucocytes into the inflamed colon and the overall pathophysiology of ulcerative colitis.

Alicaforsen is a 20 base phosphorothioate oligodeoxynucleotide designed to specifically hybridise to a sequence in the $3^{\prime}$-untranslated region of human ICAM-1 (CD54) messenger RNA. RNase-H, a ubiquitous family of nucleases, uniquely recognises oligodeoxynucleotide-RNA heterodimers and will destroy the RNA strand of such heterodimers by enzymatic cleavage. ${ }^{19}$ A highly specific reduction in ICAM-1 mRNA, and consequently ICAM-1 expression, follows and has been demonstrated in human endothelial cell culture models, in mouse heterotopic heart transplant models, in mouse pancreatic islet transplant models, in rat heart and kidney transplants, and in mouse models of colitis. ${ }^{20} 21$

A previous study of ISIS 2302 administered intravenously ${ }^{21}$ failed to show a treatment effect in patients with active steroid dependent Crohn's disease. Post hoc pharmacokinetics and population analysis of these results suggested a correlation between drug exposure and response to ISIS 2302. This hypothesis is being further explored in additional randomised controlled clinical trials in Crohn's patients. In the current study, ISIS 2302 was administered topically. It may be that topical administration is a more effective way of administering antisense to the target tissue in inflammatory bowel disease.

The alicaforsen enema doses used in this study $(60 \mathrm{ml}$ of $0.1,0.5,2$, or $4 \mathrm{mg} / \mathrm{ml}$ ) were selected based on the safety and efficacy demonstrated with comparable doses in murine models of colitis (data on file at Isis Pharmaceuticals). As alicaforsen bioavailability from inflamed colon in humans was not known, a conservative dose escalation protocol was used. Dose duration (daily evening dose for 28 consecutive days) was selected to evaluate duration of therapy comparable with other anti-inflammatory therapies for ulcerative colitis.

Retention enemas are an effective way of achieving therapeutic concentrations within the mucosa of the distal and left colon. Meta-analyses of mesalamine therapy in ulcerative colitis have reported that topical mesalamine therapy is superior to oral therapy for induction and maintenance of remission. ${ }^{12}$ Enema therapy is well tolerated by patients with ulcerative colitis and has become an accepted route of drug administration. ${ }^{22}$

In this study, all four alicaforsen dosing regimens resulted in high, dose dependent, tissue concentrations in mucosal biopsy samples of colon obtained on day 29. Median tissue concentrations ranged from 0.48 to $5.49 \mu \mathrm{g} / \mathrm{g}$, and patient compliance with administration of the retention enemas for 28 consecutive days was excellent (only one patient received less than 27 doses).

In conclusion, alicaforsen enema demonstrated acute and long term improvement in DAI and resulted in complete endoscopic remissions in a significant percentage of patients treated with drug concentrations of $2 \mathrm{mg} / \mathrm{kg}$ or $4 \mathrm{mg} / \mathrm{kg}$. The drug was well tolerated with no major safety issues identified. These early findings should be validated in additional larger, prospective, randomised, controlled, clinical trials.

\section{ACKNOWLEDGEMENTS}

This clinical trial was funded by Isis Pharmaceuticals, Carlsbad, California, USA. The authors appreciate the assistance of Chris Ward, $\mathrm{MA}$, of Isis Pharmaceuticals, in the preparation of this manuscript.

\section{Authors' affiliations}

S J H van Deventer, Academisch Medisch Centrum, Amsterdam, the Netherlands

J A Tami, M K Wedel, Isis Pharmaceuticals, Carlsbad, California, USA Conflict of interest: Drs Tami and Wedel are employees of Isis Pharmaceuticals.

\section{APPENDIX}

Members of the European Colitis Study Group include: A Wiercinska-Drapalo, Oddizial Chorob Zakazynch, Zurawia, Poland; P Vergauwe, St Niklaas Hospital, Kortrijk, Belgium; J Bogdal, Uniwersytet Jagiellonski Collegium Medicum, Krakow, Poland; G D’Haens, Immeldaziekenhuis, Bonheiden, Belgium; M Klopocka, Wewnetrznych Akademii Medycynej Biziel, Bydgoszcz, Poland; C Plane, Centre Hospitalier Germon and Gauthier, Bethune, France; G Rydzewska, Centralny Szpital Kliniczny Ministerstwa, Warsaw, Poland; J F Colombel, Universite de Lille, France; J L Dupas, CHRU d'Amiens, France; A Boruchowicz, Centre Hospitalier, Valenciennes, France.

\section{REFERENCES}

1 Cohen RD, Woseth DM, Thisted RA, et al. A meta-analysis and overview of the literature on treatment options for left-sided colitis. Am J Gastroenterol 2000;95:1263-76.

2 Marshall JK, Irvine EJ. Putting rectal 5-aminosalicylic acid in its place: the role in distal ulcerative colitis. Am J Gastroenterol 2000;95:1628-36.

3 Dustin ML, Rothlein R, Bhan AK, et al. Induction by IL-1 and interferon: tissue distribution, biochemistry, and function of a natural adherence molecule (ICAM-1). J Immunol 1986;137:245-54.

4 Rothlein R, Dustin ML, Marlin SD, et al. A human intercellular adhesion molecule (ICAM-1) distinct from LFA-1. J Immunol 1986;137:1270-4.

5 Simmons D, Makgoba MW, Seed B. ICAM, an adhesion ligand of LFA-1, is homologous to the neural cell adhesion molecule NCAM. Nature 1988;331:624-7

6 Marlin SD, Springer TA. Purified intercellular adhesion molecule-1 (ICAM-1) is a ligand for lymphocyte function-associated antigen-1 (LFA-1). Cell 1987:51:813-19.

7 Diamond MS, Staunton DE, deFougerolles AR, et al. ICAM-1 (CD54): a counter-receptor for Mac-1 (CD1 1b/CD18). J Cell Biol 1990;1 11:3129-39.

8 Butcher EC. Leukocyte-endothelial cell recognition: Three (or more) steps to specificity and diversity. Cell 1991;67:1033-6.

9 Furie MB, Tancinco MCA, Smith CW. Monoclonal antibodies to leukocyte chemoattractant-stimulated neutrophil transendothelial migration in vitro. Blood 1991;78:2089-97.

10 Oppenheimer-Marks N, Davis LS, Bogue DT, et al. Differential utilization of ICAM-1 and VCAM-1 during the adhesion and transendothelial migration of human T lymphocytes. J Immunol 1991; 147:2913-21.

11 Van Aasche G, Rutgeerts P. Antiadhesion molecule therapy in inflammatory bowel disease. Inflamm Bowel Dis 2002;8:291-300.

12 Bennett CF, Kornbrust D, Henry S, et al. An ICAM-1 antisense oligonucleotide prevents and reverses dextran sulfate sodium-induced colitis in mice. Gastroenterology 1998;114:1133-42.

13 Taniguchi T, Tsukada H, Nakamura H, et al. Effects of the anti-ICAM-1 monoclonal antibody on dextran sodium sulphate sodium-induced colitis in rats. J Gastroenterol Hepatol 1998;13:945-9.

14 Schroeder KW, Tremaine WJ, Ilstrup DM. Coated oral 5-aminosalicylic acid therapy for mildly to moderately active ulcerative colitis. N Engl J Med 1987;317:1625-9. 
15 Truelove SC, Witts U. Cortisone in ulcerative colitis. BMJ 1955:2:1041-8.

16 Yu RZ, Baker B, Chappell A, et al. Development of an ultrasensitive noncompetitive hybridization-ligation enzyme-linked immunosorbent assay for the determination of phosphorothioate oligodeoxynucleotide in plasma. Anal Biochem 2002;304:19-25.

17 Vainer B, Nielsen $\mathrm{OH}$. Changed colonic profile of P-selectin, plateletendothelial cell adhesion molecule-1 (PECAM-1), intercellular adhesion molecule-1 (ICAM-1), ICAM-2, and ICAM-3 in inflammatory bowel disease. Clin Exp Immunol 2000;121:242-7.

18 Vainer B, Sorenson S, Nielsen $\mathrm{OH}$, et al. Subcellular localization of intercellular adhesion molecule-1 in colonic mucosa in ulcerative colitis. Ultrastruct Pathol 2002;26:113-21.
19 Wu H, Lima WF, Crooke ST. Properties of cloned and expressed human RNase H1. J Biol Chem 1999;274:2870-8.

20 Bennett CF, Condon T, Grimm S, et al. Inhibition of endothelial cell-leukocyte adhesion molecule expression with antisense oligonucleotides. J Immunol 1994; 152:3530-40.

21 Bennett CF, Kornbrust D, Henry S, et al. An ICAM-1 antisense oligonucleotide prevents and reverses dextran sulfate sodium-induced colitis in mice. $J$ Pharmacol Exp Ther 1997;280:988-1000.

22 Yacyshyn BR, Chey WY, Goff J, et al. Double blind, placebo controlled trial of the remission inducing and steroid sparing properties of an ICAM-1 antisense oligodeoxynucleotide, alicaforsen (ISIS 2302), in active steroid dependent Crohn's disease. Gut 2002;51:30-6.

\section{EDITOR'S QUIZ: GI SNAPSHOT}

\section{An important piffall in diagnosing gall bladder cancer}

Robin Spiller, Editor

\section{Clinical presentation}

A 72 year old male was admitted for recurrent right upper abdominal pain, intermittent fever, and $12 \mathrm{~kg}$ weight loss over a period of six months. Some liver enzymes were elevated (that is, alkaline phosphatase $10.14 \mu \mathrm{mol} / \mathrm{l} / \mathrm{s}$ (norm 1.77-4.4), gamma glutamyl transferase $7.81 \mu \mathrm{mol} / \mathrm{l} / \mathrm{s} \quad(0.18-$ $0.83)$ ). CA 19-9 was within the normal range. Abdominal ultrasound revealed cholestasis, a thickened gall bladder wall, and suspected bile duct stones. Endoscopic retrograde cholangiography showed a proximal common bile duct and hilar stenosis, highly suspicious of gall bladder or bile duct cancer. Biopsies were taken showing dysplastic epithelium. Contrast enhanced ultrasound, magnetic resonance imaging, and computed tomography scanning were performed, revealing a hilar infiltration $(34 \mathrm{~mm})$ and a thickened gall bladder wall compatible with gall bladder cancer (fig 1A-C). Positron emission tomography (PET) with $\left[{ }^{18} \mathrm{~F}\right]$ fluoro-2-deoxy-Dglucose (FDG-PET) identified two metabolically active areas in the region of the gall bladder and the hilus (fig ID). Taken together, a diagnosis of gall bladder cancer was made. Due to comorbidities and local tumour extension, the patient was treated with palliative insertion of plastic bile duct stents.

\section{Question}

What is the most probable clinical course of the patient?

See page 1664 for answer

This case is submitted by:

J Feisthammel, K Caca, J Mössner

Department of Internal Medicine II, University of Leipzig, Leipzig, Germany T Kahn Department of Radiology, University of Leipzig, Leipzig, Germany R Kluge

Department of Nuclear Medicine, University of Leipzig, Leipzig, Germany

Correspondence to: Professor K Caca, Department of Internal Medicine II, University of Leipzig, Philipp-Rosenthal-Str 27, 04103 Leipzig, Germany; caca@medizin.uni-leipzig.de

doi: $10.1136 /$ gut.2003.036947
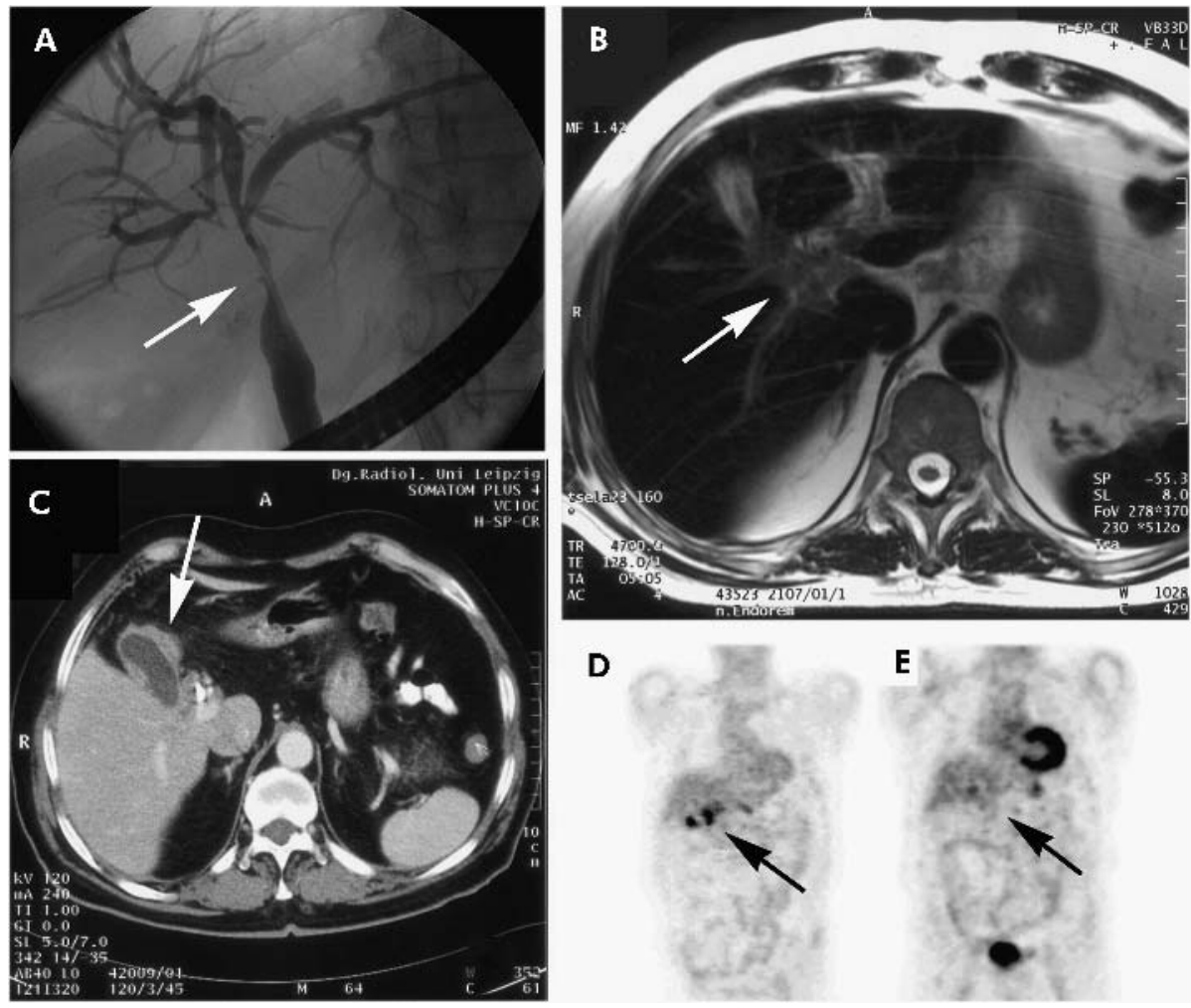

Figure 1 Endoscopic retrograde cholangiography (A), magnetic resonance imaging (B), and computed tomography scanning (B) of the gall bladder, and [ $\left.{ }^{18} \mathrm{~F}\right]$ fluoro-2-deoxy-D-glucose positron emission tomography (D, E). 